\title{
Gender Differences Among Children in Language Expression of Emotional and Motion Related Words
}

\author{
Jiaying Xu ${ }^{1, a}$, Feiqi Lin ${ }^{2, b}$, Shuning Liu $^{3, \mathrm{c}}$, Xiying Peng ${ }^{4, \mathrm{~d}}$, Ziqi Wei ${ }^{5, \mathrm{e}}$ \\ ${ }^{1}$ Department of Psychology, The University of Texas at Austin, Austin, Texas, United States \\ ${ }^{2}$ Grand Rapids Christian High School, Grand Rapids, Michigan, United States \\ ${ }^{3}$ Wuhan Britain-China School, Wuhan, Hubei, China \\ ${ }^{4}$ Teaching Chinese to Speakers of Other Languages, Shenzhen University, Shenzhen, Guangdong, China \\ ${ }^{5}$ Vanke Meisha Academy, Shenzhen, Guangdong, China \\ a jasmine142317@utexas.edu \\ b22linvi@grchristian.org \\ c1296453043@qq.com \\ d1634651123@qq.com \\ ${ }^{e}$ weiziqi@stu.vma.edu.cn
}

\begin{abstract}
Gender differences exist in language expressions among adults and yet, there are different kinds of words in language expression and limited research was done focusing on children. This research proposal is about gender differences among children. We hypothesized that girls will use more emotional words in their talking and boys will use more motion-related words. We intend to recruit 50 children and ask them to describe a series of photos which a recording device equipped on them. Using the recording, researchers will calculate the frequency of emotional and motion-related words to find out whether gender differences exist in expressing these two types of words. The results can help and apply to the education or child psychology field where gender differences can be embedded into teaching techniques.
\end{abstract}

Keywords: children, gender differences, language learning, emotional words, motion related words

\section{INTRODUCTION}

The debate over how men and women differ in language expression is far from settled. Indeed, we see those differences in our daily life, forming the stereotype that women tend to express themselves more emotionally and men tend to express more rationally [1]. Such stereotypes put vastly different pressures on men and women as they navigate their roles in society. For example, women's expressions are more often constrained by conformity to feminine characteristics such as displaying motherly love, weakness, domesticity, etc. while men's expressions are expected to represent power, dominance, and emotional restraint. These traits in language expressions lead to significant gender differences in the workplace, education, social activities, and household environment. Since available studies have already investigated the topic of gender differences in language expression among adults, it is possible that gender differences exist within a younger age cohort, reinforcing gender differences in different aspects such as stereotypical characteristics, work performance, and roles in our culture and society. Moreover, compared to adults, language learning is more important to young children because language can help children to communicate with adults. Looking into the gender differences among language expressions might help researchers to find out whether these differences developed through the language learning process or due to other factors in later developmental periods. Inspired by these thoughts, this study is focusing on gender differences in children's language expression, specifically in emotional and motion-related words.

\section{BACKGROUND}

Several earlier studies demonstrate that men and women have similar emotional experiences, but they do differ in related word expressions. Researchers such as McRae et al. used fMRI signals from the amygdala to measure gender differences in emotional reactivity by 
comparing different genders' responses to negative and neutral pictures, where negative pictures elicit people's negative emotional responses and neutral pictures will not cause emotional changes for people [2]. Researchers later found out that males and females did not differ in brain responses, which supports few differences that appear in the perception of men's and women's emotional experiences. However, researchers identified that gender differences do exist in language expression. For example, Sells et al. investigated gender and environmental differences in experiencing and expressing emotion [3]. They recorded 47 participants' verbal responses after watching an emotionally provocative video in both public and private settings. The result indicates that women used more emotional words than men, regardless of the experiment setting.

Differ from women, we hypothesis that men tend to express some particular kinds of words such as motionrelated words. First, characteristics of language expression show great gender differences: while women's speech has been described as emotional and uncertain, men's speech (in tone and word choice), in contrast, is direct and rational [4]. For instance, women might say: "Shall we close the door?", which shows consideration of others' emotions; but men might say: "Close the door, please.", which is more direct and less emotional. Such differences suggest that men's speech is more direct and less emotional than women's. Thus, we believe that motion-related words referring to exact happenings will be used more in men's speech. Besides, men are more interested in sports and exciting activities which are based on motion words, so the usage of words and terms concerning these fields is higher [5]. In neuroscience studies related to language expression, Ullman et al. indicated that women tend to rely on declarative memory in language processing, but men tend to rely on procedural memory, including the memory of motion, to conduct real-time language processing $[6,7]$. According to this memory processing difference, chances are that men perform motion words ahead of emotional words in most situations.

Evidence from previous studies has shown that women tend to express more emotional words (e.g., love, sad, happy) than men, and men might have a greater tendency to express motion-related words (e.g., walk, jump, ride) in many contexts. Nevertheless, these studies have limitations. First, these findings were largely based on experiments designed for adults, and few studies were conducted to examine the gender differences in language expression of children. Therefore, it is difficult to determine whether such differences in emotional words and motion words appear earlier in childhood, are developed from childhood to adulthood, or appear in other developmental periods. Second, some studies indicated that females have a general advantage in language which might explain the better performance in emotional-related word expressions. For example, Eriksson et al. concluded that girls showed a small but highly reliable word production advantage [8]. Still, such conclusions are so broad that researchers overlooked the possibility of males' advantages in word production of specific domains. Therefore, this study will focus on two distinct domains - emotional words and motion words, to do further and detailed research on gender differences in children's language expression.

\section{PROPOSED STUDY}

The proposed study aims to determine whether boys and girls have different frequencies in expressing words from two distinct domains: emotional-related words and motion-related words. Children will be asked to describe a series of pictures that include emotional and motionrelated content, and researchers will calculate the frequencies of the emotional and motion-related words in the overall words said by children. This study will have four alternative results: First, girls express emotional words more frequently, and boys express motion words more frequently; Second, girls show use both words more frequently than boys; Third, only one group (emotional Vs. motion) shows a significant difference; Lastly, both boys and girls have almost the same frequencies in expressing emotional words and motion words, so no significant differences were found in the study.

\section{METHOD}

\subsection{Participants}

As Sells et al. recorded 22 boys and 25 girls in their experiment, we choose $\mathrm{N}=25$ boys and $\mathrm{N}=25$ girls ( $\mathrm{N}$ $=50$ in total) as our participants to ensure the experiments' reliability [3]. According to the results from wordbank (Figure 1-2), only about $10 \%$ of 30-month-old children have the ability to produce 600 English words, which includes the most common motion and emotion words [9]. Hence to ensure all children we test are able to express most of the words, we choose participants from 4 to 5 years old. In order to test our confounding variables, such as environments, parents' ages, parents' careers, and parents' educational background into consideration, parents will also participate in filling in a survey during the experiment. 


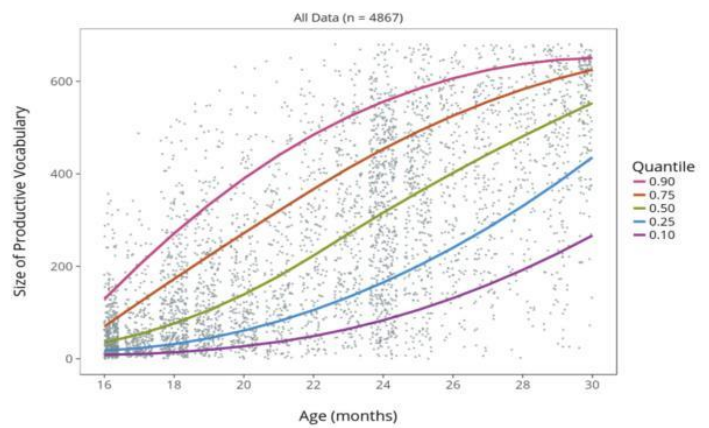

Figure 1 Productive English vocabulary size of children from 16-30-months old

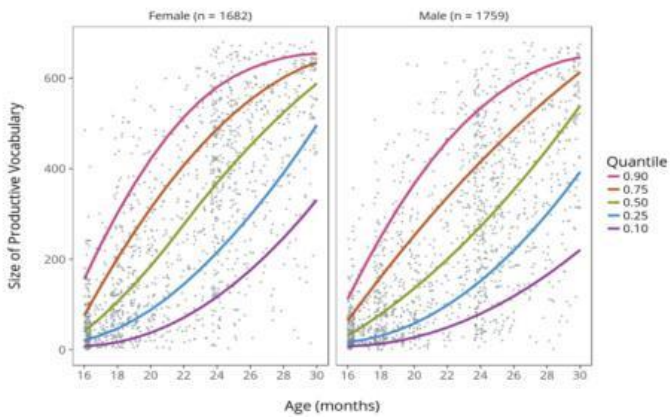

Figure 2 Size of productive English vocabulary in different genders

\subsection{Materials}

The study will have 10 photos, and each contains a figure presenting a motion with an emotional facial expression (Figure 3 shows an example), such as a person running with sad emotion or a person who is swimming with happy emotion. Instructions will be pre-recorded through a recording device, and during the experiment, the researchers will play the pre-recorded instructions such as "what can you see from this photo" to ensure each participant receive the message from the same person in the same volume and tone. Our confounding variable will be about children's home environment, such as numbers of siblings, and parents will receive the 2021 American Community Survey, which is a survey created by United States Census Bureau. The questionnaire includes parents' ethnicity, occupation, education level, marital status, housing situation, etc. [10]. Lastly, a surprise gift, cotton candy, will be prepared and give to children after they complete the experiment.

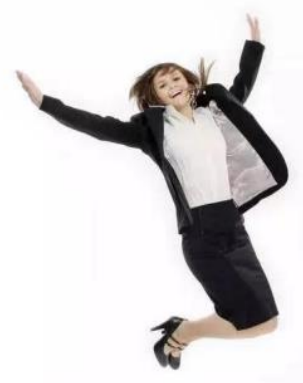

Figure 3 Example of photos used in the experiment: A woman who is jumping while expressing a happy emotion.

\subsection{Design}

The entire experiment will be held in a classroom where children will feel comfortable talking, and there will only be one participant for each trial in the room. The researcher will manipulate everything remotely, including start playing the pictures through a projector and start the recording devices for instructions. The prerecorded instructions will include one statement that children will receive surprise if they do what the recording says and stay in their seats through the experiment and the purpose of this statement is to use children's desires to surprise gift to make sure they complete the experiment without a researcher physically be in the room with them. Each trial will have 10 pictures switched automatically with the 1-minute time interval, and the instructions will be played simultaneously. This design ensures that the entire experiment can be carried out without being disturbed, and the authenticity and accuracy of the experiment can be guaranteed. While the children are doing experiments, the parents will complete the 2021 American Community Survey in the next room [10]. The content of the questionnaire includes race, family members, parent's education level, and occupation. Through this survey, it is possible to know in advance the differences between the participants' living environment and education environment. The dependent variable is the frequency of how often children produce emotional and motion-related words. The independent variables are the gender of children. 


\subsection{Procedure}

Before starting the experiment, the participant will be taken into a classroom by the researcher, and the researcher will remain quiet during this process. After the researcher leaves, the recording device will start to play the pre-recorded instructions including telling participants there will be a surprise gift for them if they can stay in seats and follow the instruction for 10 minutes and guiding the participant to observe the pictures. Each group of pictures will be displayed for one minute with no repeat. For each picture, the recording will be played repeatedly to guide the children to describe the picture's content, and another recording device will record everything the participant said during the experiment. When children are describing pictures in the experiment room, their caregivers will be sitting in the next room and filling out a survey that also lasts about 10 minutes. The results of children's description will be supported by the frequency of emotional and motion-related words, and the frequency will be based on the ratio of emotional and motion words that appeared in the overall words expressed by participants individually. Researchers will also look into the socioeconomic factors to find out if any significant differences exist in the results.

\section{RESULTS AND DISCUSSION}

Our study will be focusing on the gender differences in language expression, which will have 5 possible results presenting in Figure 4-8. If our results show both significant differences between boys and girls in emotional and motion-related words (Figure 4), it indicates that girls are using emotional-related words more frequently than boys, and boys have a higher usage of motion-related words compared to girls. This result would prove that the difference is developed through early childhood, and also gender differences do exist in language expression, but it is not due to a single-gender advantage. The second possible result is that girls will show higher usage in both emotional and motion-related words which might contribute to the conclusion that girls might have an overall language advantage over boys (Figure 5). The third and fourth possibilities are that either one of the emotional or motion-related words shows a significant gender difference, so that means gender differences do exist in certain kinds of words but do not apply to the overall language expression (Figure 6-7). However, if the result shows non-significant differences (Figure 8), it proves that the gender difference is not showing until later childhood.

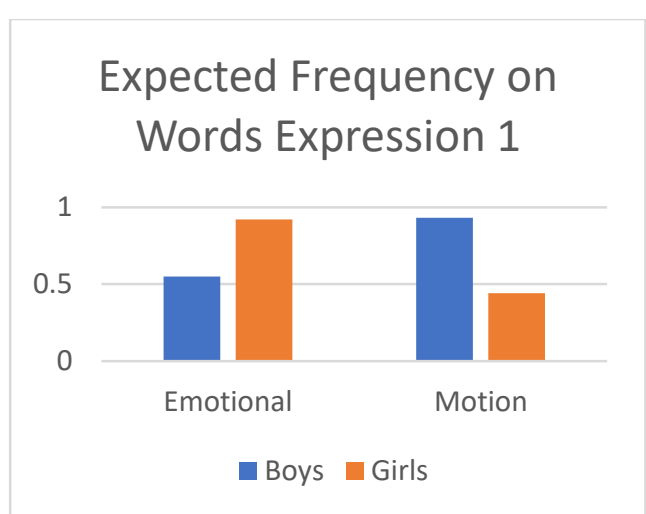

Figure 4 Expected Frequency on Words Expression 1

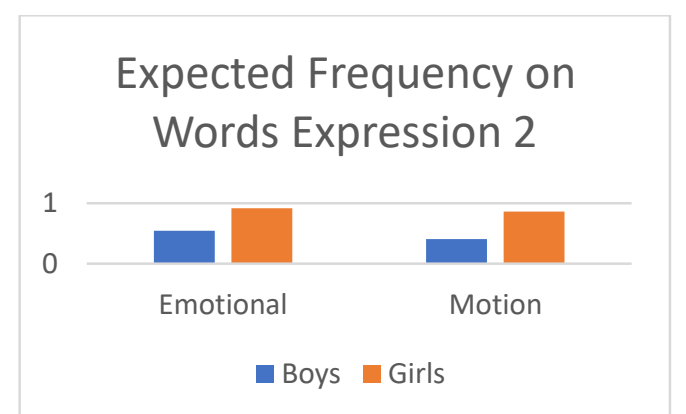

Figure 5 Expected Frequency on Words Expression 2

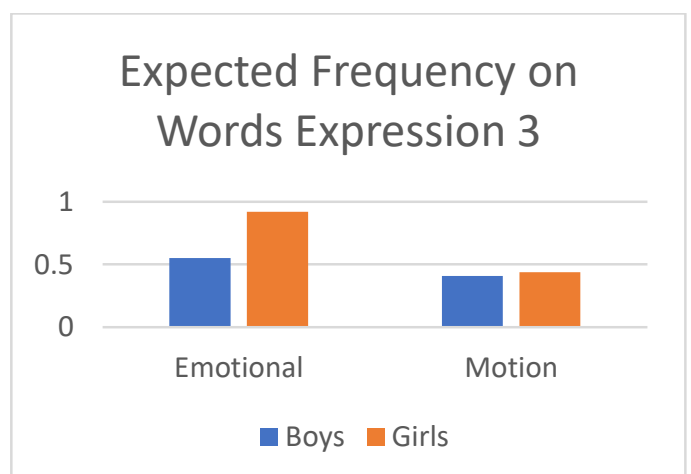

Figure 6 Expected Frequency on Words Expression 3

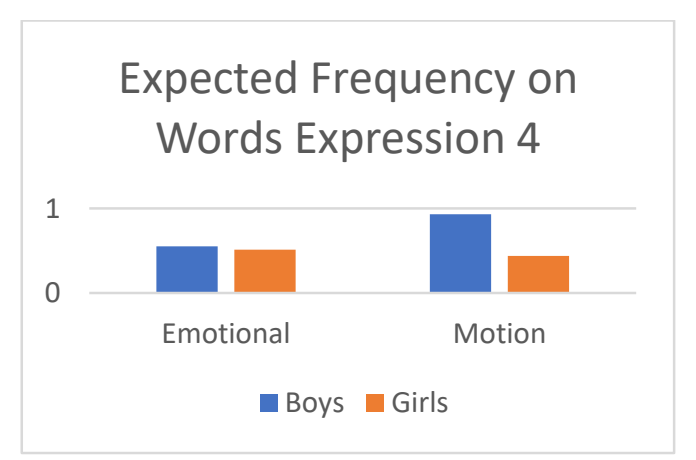

Figure 7 Expected Frequency on Words Expression 4 


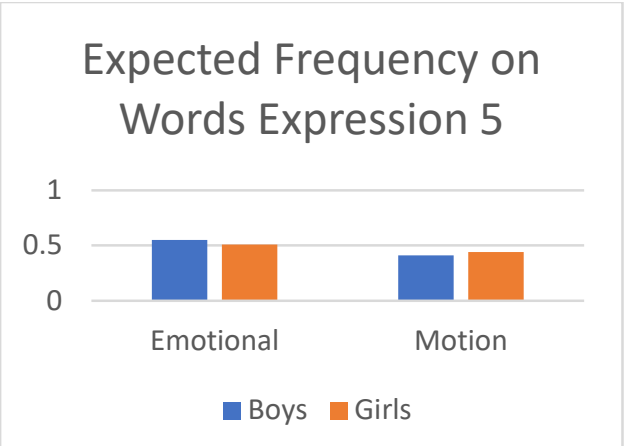

Figure 8 Expected Frequency on Words Expression 5

\section{CONCLUSION AND FUTURE DIRECTIONS}

Some limitations exist in this study. First, it remains unclear whether this difference is due to biological factors, which means children are born with this difference, or due to social and environmental factors, or because of a different understanding of the different types of words. This also leads to our second limitation which is that the study does not solve whether there are gender differences in word comprehension, or the differences are only presented in word expressions. If there are gender differences in understanding the words, girls might have a deeper understanding of emotional words, and boys might have a stronger understanding of motion-related words. Third, our study only focuses on emotional and motion-related words, and there are still many other kinds of words existing in English, so we cannot conclude there is a gender difference in the overall language expression.

Researchers can test on more different labels of words to find out the gender differences in overall language expression for future directions. We picked emotional and motion-related words from the research did on adults. And those research results suggest that women will tend to use emotional words and men will tend to use motionrelated words. In the future, Researchers can observe children directly to see children's own unique labels rather than acquire those labels from adults' language expression because there might be changes during developmental periods.

Researchers can also look into the mechanisms behind the phenomena shown in this research. In this research, we can only suggest that there are gender differences exist in language expressions. However, expression is not the front of the talking process. Before expression, thinking is involving, and understanding might also play a role inside. Future research should look into different stages in the talking process to understand when exactly the gender differences happen. if future research can provide evidence that boys and girls are showing differences in language learning, this result might be able to be further utilized in child psychology and education. For child psychology, gender differences can be used as a technique to elicit children to express themselves more when children are shy or reluctant to communicate. In education, for children who are learning a second language, teachers might be able to apply gender difference into teaching, which will help children to learn more efficiently. For example, if teachers focus more on teaching boys emotional words and girls on motion words, it might help boys and girls better perform second language expression or if teachers use emotional stimulus to arouse girls' learning motivation and motionrelated stimulus to arouse boys' learning motivation, children might have a better performance in language expressions. Another advantage is that it might also enable people to get a better understanding of different characteristics of language expression between genders so that they can do better intergender communication.

In conclusion, gender differences in language expressions among children is a topic that has limited research. It is crucial to do research in this field and understand the mechanisms behind these phenomena. Learning this field can help both adults and children since parents might able to learn how to better communicate with their children, and children might receive better education or stronger language ability.

\section{REFERENCES}

[1] Fabes, R. A., \& Martin, C. L. (1991). Gender and age stereotypes of emotionality. Personality and Social Psychology Bulletin, 17(5), 532-540. https://doi.org/10.1177/0146167291175008

[2] McRae K, Ochsner KN, Mauss IB, Gabrieli JJD, Gross JJ. Gender Differences in Emotion Regulation: An fMRI Study of Cognitive Reappraisal. Group Processes \& Intergroup Relations. 2008;11(2):143-162. https://doi.org/10.1177/136843020708803

[3] Sells, David J.; Martin, Randal B. (2001). Gender and Modality Differences in Experiencing and Emotional Expression. Canadian Journal of Counselling, 88-176. https://eric.ed.gov/?id=EJ629090

[4] McConnell-Ginet, S. \& Borker, R. \& Furman, N. (eds.) 1980. Women and Language in Literature and Society. New York: Praeger Publisher

[5] Shao, Meng. (2008). Gender Differences in InterGender Communication - An Empirical Study (Master Dissertation, Ocean University of China). https://kns.cnki.net/KCMS/detail/detail.aspx?dbna $\mathrm{me}=\mathrm{CMFD} 2009 \&$ filename $=2008176535 . \mathrm{nh}$

[6] Ullman, M. T., Estabrooke I. V., Steinhauer, K., Brovetto, C., Pancheva, R., Ozawa, K., et al. (2002). Sex differences in the neurocognition of language. Brain and Language, 83, 141-143 
[7] Ullman, M. T., Miranda, R. A., \&Travers, M. $\mathrm{L}(2008)$. Sex differences in the neurocognition of language. In J. B. Becker, K. J. Berkley, N. Geary, E. Hampson, J. Herman, \& E. Young (Eds.), Sex on the brain, From genes to behavior, 291-310. https://doi.org/10.1093/acprof:oso/9780195311587. 003.0015

[8] Mårten Eriksson, Peter B. Marschik, Tiia Tulviste, Margareta Almgren, Miguel Pérez Pereira, Sonja Wehberg, Ljubica Marjanovič-Umek, Frederique Gayraud, Melita Kovacevic, Carlos Gallego (2012). Differences between girls and boys in emerging language skills: evidence from language communities. British Journal of Developmental Psychology, 30, 326-343. https://doi.org/10.1111/j.2044-835x.2011.02042.x

[9] Braginsky, M. (n.d.). Wordbank. http://wordbank.stanford.edu/

[10] Bureau, U. S. C. (2020, December 16). English Questionnaire archive. https://www2.census.gov/programssurveys/acs/methodology/questionnaires/2021/ques t21.pdf. 